probabilities and kernels, and recurrence. For a short text this is a good decision; the probabilisttechnician will find this a helpful manual about a very useful tool. But as with all manuals it is better used than read. The reader will find that the abstractions and general treatment come to life when confronted by specific application to particular Markov chains.

One application of these ideas (not covered in the book) is found in stochastic differential geometry. Consider a Brownian motion on a compact Riemannian manifold. Many workers have investigated the way in which the Brownian path wraps itself round the manifold, in homotopy or in homology. Various approaches have been adopted; one of these (exploited to great effect by Lyons and Sullivan, 1984) uses a coupling similar to the one described above. The Brownian motion is sampled whenever it visits a particular small sphere; the Markov chain is made up of the position of the Brownian motion on the sphere together with the homotopy type of its most recent excursion through the whole manifold (to make this precise one needs the notion of the local time on the sphere of the Brownian motion). The result is almost a random walk on the homotopy group of the manifold. However to obtain a Markov property one must employ information on where the Brownian motion last hit the small sphere. This moves the problem into the province of Markov chains on uncountable state space. It should be plain from the brief description here how coupling methods can be applied. One can establish estimates on harmonic measure and thus obtain bounds (of the form given above) on the transition kernel.

W. S. KENDALL

\title{
REFERENCES
}

W. Doeblin (1938), Exposé de la théorie des chaînes simples constantes de Markoff à un nombre fini d'etats, Rev. Math. de PUnion Interbalkanique 2, 77-105.

T. Lyons and D. Sullivan (1984), Function theory, random paths and covering spaces, J. Differential Geom. 19, 299-323.

\section{Berndt, Bruce C. Ramanujan's Notebooks, Part I (Springer-Verlag, 1985), x + 357 pp. DM 188.}

During the years before he came to Cambridge in 1914 the distinguished Indian mathematician Srinivasa Ramanujan filled three notebooks with mathematical formulae and theorems, most of them being stated without proof. The third and shortest of the notebooks consists of approximately 100 pages of unorganized material. The second, consisting of 252 pages divided into 21 chapters, is a revised and enlarged edition of the first notebook and is thus the fullest and most interesting of the three. At the time when G. H. Hardy and B. M. Wilson were preparing Ramanujan's collected papers for publication by the Cambridge University Press it had been intended to publish the notebooks as an appendix. However, this proposal was abandoned, largely for financial reasons, but possibly also because Hardy realized (as a result of his excessive labour in preparing chapter 12 of the first notebook for publication) that to accomplish the necessary editorial work satisfactorily was a mammoth task likely to extend over many years.

By the mid twenties, however, G. N. Watson of Birmingham had begun, in collaboration with a colleague, to write a number of papers on "Theorems stated by Ramanujan" and, for this reason, Hardy gradually over the years passed on to him all his Ramanujan material. Watson and B. $\mathbf{M}$. Wilson divided the 21 chapters of Notebook 2 between them, Wilson taking chapters $2-13$ and Watson chapters 14-21; chapter 1, on magic squares, is of less interest, having been written when Ramanujan was a schoolboy, and containing nothing new. Both men did a great deal of work on their assigned chapters, but Wilson died suddenly in 1935 in Dundee, where he had moved from Liverpool to succeed Professor Steggall. His material was passed to Watson, whose output of work on the notebooks gradually declined and had ceased altogether by the beginning of the second world war; nevertheless he retained his interest in Ramanujan until his death in 1965. The considerable dossier of his papers found in his house after his death was passed to Trinity College, Cambridge, of which he, Hardy and Ramanujan had all been Fellows. There it lay, comparatively unnoticed, until Professor Berndt assumed the arduous task of continuing the work begun by Watson and Wilson and editing the notebook for publication. In so doing he has done a tremendous service to the mathematical world by his careful and critical appraisal of Ramanujan's 
work, and he has demonstrated his wide knowledge by placing it in a historical setting, finding, for example, that in some instances Ramanujan had been anticipated by classical writers, while in others - as for the Bell polynomials - he had obtained results later discovered by others.

The present volume is the first of three. It covers chapters 1-9 of the second notebook as well as the three quarterly reports submitted to the University of Madras under the terms of his scholarship. The various chapters, some of them in collaboration with other authors, have previously appeared separately in mathematical journals. They comprise a total of 759 different entries. With a very few exceptions, where the intent of the entry is not clear, proofs are given under appropriate conditions, or references are given to the literature, where the results are not new. Because of his lack of an orthodox mathematical background, Ramanujan, when he came to England, had little idea of what constituted a mathematical proof and, as Littlewood wrote, "if a significant piece of reasoning occurred somewhere, and the total mixture of evidence and intuition gave him certainty, he looked no further".

Possibly because of some remarks made by Hardy, there has been an impression that much of Ramanujan's work was not new and that the disappointment supposedly suffered by him on this account had an adverse effect upon his health. There has never been any strong evidence for this opinion and, as Professor Berndt has been the first to point out, it is truly remarkable how few of the entries are results already known at the time when they were written. It is, of course, true that many are outside the main stream of 20th century mathematics, and Ramanujan was perhaps the last great producer of mathematical formulae. If he had had an orthodox upbringing he might, as Hardy wrote, have discovered more that was new and of greater importance. On the other hand, as Professor Berndt points out, his output of results might have been severely curtailed, as he might have felt inhibited about stating results unrigorously obtained.

The chapters covered in the book deal with magic squares, inverse tangent formulae, series inversion, special functions and numbers such as Eulerian polynomials and numbers, Bernoulli numbers, Riemann zeta function, gamma function, divergent series, and the transformation and evaluation of infinite series. Infinte series abound throughout; some of them are divergent, but many can be considered as asymptotic series.

From such a plethora of results it is impossible to select representative samples. I content myself by selecting one entry, which Ramanujan knew to be false as he cancelled it, but, nevertheless, gives something of the flavour of his work:

"For $|x|<1$,

$$
\prod_{k=1}^{\infty}\left(1-x^{p_{k}}\right)^{-1}=1+\sum_{k=1}^{\infty} \frac{x^{p_{1}+p_{2}+\cdots+p_{k}}}{(1-x)\left(1-x^{2}\right) \ldots\left(1-x^{k}\right)},
$$

where $p_{1}, p_{2}, \ldots$ denote the primes in ascending order."

If $c_{n}$ and $d_{n}(n \geqq 0)$ denote the coefficients of $x^{n}$ on the left and right sides, respectively, then, quite amazingly, $c_{n}=d_{n}$ for $n \leqq 20$, but $c_{21}=30$ and $d_{21}=31$.

The book, although expensive, is, as one would expect from the publishers, beautifully printed and produced.

R. A. RANKIN

UPMEIER, H. Symmetric Banach manifolds and Jordan $C^{*}$-algebras (North-Holland Mathematics Studies 104, North-Holland, Amsterdam-New York-Oxford, 1985), 444 pp. DFl. 150.

The theory of symmetric complex Banach manifolds and Jordan $\mathrm{C}^{*}$-algebras has been developing steadily over the last couple of decades. The advance has been guided by classical results for finite-dimensional manifolds and Lie groups and by parallel developments in Jordan $\mathrm{C}^{*}$-algebras closely linked to the theory of operator algebras. In this book the author studies the theory of symmetric manifolds over a Banach space and closely related areas. There are monographs on Jordan $\mathrm{C}^{*}$-algebras but not on their relationship with symmetric manifolds. This book fills an empty slot in the literature. The book is divided into two parts in a natural way. In the first part the theories of Banach manifolds over a Banach space and Banach Lie groups are 